UBCTP 92-009

\title{
Quarks as Topological Defects or What is Confined Inside a Hadron?
}

\author{
A. Kovner ${ }^{\dagger *}$ and B. Rosenstein ${ }^{\& * *}$ \\ Physics Department, University of British Columbia \\ Vancouver, B.C., Canada, V6T 1Z1
}

\begin{abstract}
We present a picture of confinement based on representation of quarks as pointlike topological defects. The topological charge carried by quarks and confined in hadrons is explicitly constructed in terms of Yang - Mills variables. In $2+1$ dimensions we are able to construct a local complex scalar field $V(x)$, in terms of which the topological charge is $Q=-\frac{i}{4 \pi} \int d^{2} x \epsilon_{i j} \partial_{i}\left(V^{*} \partial_{i} V-\right.$ c.c. $)$. The $\mathrm{VEV}$ of the field $V$ in the confining phase is nonzero and the charge is the winding number corresponding to homotopy group $\pi_{1}\left(S^{1}\right)$. Qurks carry the charge $Q$ and therefore are topological solitons. The phase rotation of $V$ is generated by the operator of magnetic flux. Unlike in $Q E D$, the $U(1)$ magnetic flux is explicitly broken by the monopoles. This results in formation of a string between a quark and an antiquark. The effective Lagrangian for $V$ is derived in models with adjoint and fundamental quarks. Baryon is described as a $\mathbf{Y}$ - shaped configuration of strings. In $3+1$ dimensions the explicit expression for $V$ and therefore a detailed picture is not available. However, assuming the validity of the same mechanism we point out several interesting qualitative consequences. We argue that in the Georgi - Glashow model or any grand unified model the photon (in the Higgs phase) should have a small nonperturbative mass. Moreover, $W^{ \pm}$should be confined although with small string tension.
\end{abstract}

$\dagger$ NSERC international postdoctoral fellow.

${ }^{\$}$ Address after September 1, 1992: Theory Division, T-8 MS B285, Los Alamos National Laboratory, Los Alamos, NM 87545.

*KOVNER@PHYSICS.UBC.CA

\&Address after October 1, 1992: Institute of Physics, Academia Sinica, Taipei 11529, Taiwan. 
**BARUCH@PHYSICS.UBC.CA 


\section{Introduction.}

Understanding of confinement in QCD is still one of the major challenges of particle physics. Confinement is a commonly held intuitive notion. It eludes, however, precise definition in the framework of QCD.

In the phenomenological quark model [1] we certainly do know what is meant by "confinement". The global color charges are confined, so that all hadrons are the $S U_{c}(3)$ singlets. However although the quark model captures some important physics, it is not derived directly from QCD. The global part of the $S U(3)$ gauge group is not a global symmetry of QCD Hamiltonian. There is no $S U(3)$ symmetry related to the gauge group in the same way the electric charge $U_{e}(1)$ symmetry is related to the $U(1)$ gauge group in QED.

In QED the global $U_{e}(1)$ symmetry generator is $Q=\int d^{d} x \psi^{\dagger} \psi$. The symmetry transformation rotates the phase of charged fields: $\psi(x) \rightarrow e^{i \chi} \psi(x)$, but does not act on vector potential $A_{\mu}(x)$. This by no means coincides with the global part of the gauge group. The latter is generated by the Coulomb constraint

$$
C(x) \equiv e \psi^{\dagger}(x) \psi(x)-\partial_{i} E_{i}(x)
$$

and acts not only on $\psi$ but also on $A_{\mu}$ at spatial infinity.

In QED the first term in the Coulomb constraint, the electric charge density, $\psi^{\dagger} \psi$ is itself a gauge invariant quantity. Therefore, the electric charge, defined as its integral, is an operator acting nontrivially on physical Hilbert space. There is no analogous construction in QCD. The Coulomb constraint 
in $\mathrm{QCD}$ is

$$
C^{a} \equiv e \psi^{\dagger} \lambda^{a} \psi-\partial_{i} E_{i}^{a}+e f^{a b c} A_{i}^{b} E_{i}^{c}=0
$$

A transformation which one is tempted to identify with global color symmetry is generated by the first and the third terms in $C^{a}$ :

$$
Q^{a}=e \int d x\left(\psi^{\dagger} \lambda^{a} \psi+f^{a b c} A_{i}^{b} E_{i}^{c}\right)
$$

However, it is easy to see, that the matrix element of $Q^{a}$ between any two physical states (i.e. states that satisfy the Coulomb constraint eq.(22)) vanishes. Indeed, $Q^{a}$ transform as an adjoint representation of the global part of the gauge $S U(3)$ group (generated by $\int C^{a}$ ). According to Wigner - Eckart theorem, $\left\langle\alpha\left|Q^{a}\right| \beta>=0\right.$ when $| \alpha>$ and $\mid \beta>$ are singlets, in distinction to the Abelian case where this problem does not arise.

Since global $S U_{c}(3)$ cannot be given a satisfactory definition, the immediate question is: what is confined inside hadrons?

The quark fields that appear in the QCD Lagrangian are not gauge invariant objects and the $S U(3)$ charge that they carry is nonzero only on nonphysical states in the "large" Hilbert space of a gauge theory. On the other hand, one would like to think about constituent quarks as about strongly interacting, but real particles. Intuitively one would like to associate some kind of a conserved "quark number" $Q$ with the constituents 1 . The notion

\footnotetext{
${ }^{1}$ The connection between these constituent quarks and the bare quarks of perturbative QCD is not obvious. The constituents presumably exist as particles at intermediate scales. At large distances they are confined. At small distances, since nonabelian theory is asymptotically free, the "bare" quarks look particle like. The constituent "quark number"
} 
of confinement then can be given a precise meaning: objects that carry the charge $Q$ interact linearly and can never be separated. This is precisely what is meant by confinement in compact QED with $Q$ beeing the electric charge.

In QCD, it is said sometimes, that the triality is the confined quantum number. Triality is a discrete physical global symmetry of YM theories with matter (quarks) in fundamental representation. This criterion is however totally inadequate to account for confinement of adjoint quarks. The mechanism of confinement is presumably the same for fundamental and adjoint quarks [2, 3]. Thus one has to look for another charge which is confined.

In this paper, we point out the existence of such a charge $Q$ in nonabelian gauge theories (see eq.(9) in the following). This $U(1)$ charge is topological, in a sense that the corresponding current is "trivially" conserved (as also is the case in QED 2]). The charge $Q$ is defined in any dimensionality. In $2+1$ dimensions one can go further. Q can be represented as the winding number of a gauge invariant local complex field $V$. When the (low energy) theory is rewritten in terms of this vortex field $V$, the (gauge invariant) quarks are identified as topological solitons. An explicit topological representation of

we have in mind does not have therefore much to do with the "global color" which appears as a perturbative symmetry of bare quarks in the extreme ultraviolet limit. Throughout the paper we reserve the term "quarks" for these constituent objects. Terms like "adjoint quarks" should be understood as constituent quarks in a theory with matter fields in adjoint representation. Since we will not be discussing the ultraviolet limit, we will not be dealing with the bare quarks at all.

${ }^{2}$ In QED the current $J_{\mu}$ is a curl of a local gauge invariant quantity $\tilde{F}_{\mu \nu} \equiv \frac{1}{2} \epsilon_{\mu \nu \rho \sigma} F^{\rho \sigma}$. Physically, this means that electric charge can be measured performing local measurements at spatial infinity (Gauss law). 
$Q$ allows one to understand confinement of quarks as linear confinement of topological defects. We are able to derive explicitly the effective Lagrangian in the region of parameter space where perturbatively a nonabelian gauge theory is in a partially broken Higgs phase. Nonperturbatively it is known that the confinement and Higgs phases are analytically connected and confinement is the property of both. Although it is not a priori clear that the confined constituents are the same in both phases it is likely to be the case. We will suggest how the effective dual Lagrangian is modified in the confinement regime. The main quantitative difference between the picture of confinement in the Higgs and confinement regimes is that in the former case the string tension is associated with a mass of a pseudoscalar particle (the massive photon) while in the latter case with the mass of the lightest scalar glueball. The picture of quarks as topological defects in $3+1$ dimensions is still lacking. We will briefly return to this point in section 5 .

In this paper we investigate properties of $Q$ in $2+1$ dimensional nonabelian gauge theories. The charge $Q$ is analogous in many respects to the electric charge in QED. It was shown in [4 that in QED there exists a local gauge invariant scalar complex field, $V$, in terms of which the electric current has a standard form of the topological current associated with the homotopy group $\pi_{1}\left(S^{1}\right)$ :

$$
J_{\mu}=-\frac{i}{4 \pi} \epsilon_{\mu \nu \lambda} \partial^{\nu}\left(V^{*} \partial^{\lambda} V-\text { c.c. }\right)
$$

$V(x)$ has a physical meaning in scalar QED as a field interpolating Nielsen- 
Olesen vortices in the Higgs phase. In the Coulomb phase $V$ acquires a nonvanishing expectation value. The manifold of degenerate vacua is $S^{1}$. The degeneracy is due to spontaneous breakdown of the magnetic flux symmetry $U_{\Phi}(1)$ generated by $\Phi=\int d^{2} x B(x)$ [4]. The electric charge has the form

$$
Q=-\frac{i}{4 \pi} \int \epsilon_{i j} \partial_{i}\left(V^{*} \partial_{j} V-\text { c.c. }\right)
$$

and is the winding number of the phase of $V(x)$. A typical configuration of $V(x)$ which represents a charged particle located at point $x$ is a "hedgehog" centered at $x: V(y)=e^{i \Theta(y-x)}$.

In $2+1$ dimensional nonabelian gauge theories considered in this paper a local vortex field $V$ was constructed by t'Hooft [5]. We will show that the topological charge $Q$ carried by quarks, when expressed in terms of this field has precisely the form of eq.(55).

A simple classical picture of linear confinement emerges when we consider an effective Lagrangian for the field $V$. In the models we consider this dual Lagrangian is derived explicitly in the dilute monopole gas approximation. This dual Lagrangian is completely gauge invariant, and the interpretation therefore is given directly in terms of physical observables. The operator $V$ has a nonvanishing VEV as in QED. The main difference between abelian and nonabelian gauge theories is that the $U(1)$ vacuum degeneracy of QED is lifted in QCD. The reason is that the continuous flux symmetry in QCD is

\footnotetext{
${ }^{3}$ The massless photon is the Goldstone boson that corresponds to spontaneous breakdown of $U_{\Phi}(1)$.
} 
explicitly broken. f The monopole contribution to the dual Lagrangian is a symmetry breaking term of the type $V^{*}+V$. In the presence of such a term the topological solitons (quarks) are linearly confined. The reason is that now the theory does not have a continuum of degenerate vacua and the field $V$ at infinity must be as close to its VEV as possible. A topologically nontrivial configuration must contain a wind of the phase of $\mathrm{V}$. It is energetically favorable to perform this wind inside a strip of finite width (Fig. 1) rather than create a hedgehog - like configuration where $V \neq<V>$ everywhere in space. The formation of a string which carries a finite energy density per unit length leads to linear confinement. A soliton and an antisoliton are therefore bound by linear potential into a meson. The mechanism of confinement is the same as for linear confinement of defects in condensed matter systems [6]. The definition of $V$ and the derivation of the effective Lagrangian in the Georgi-Glashow model and its $S U(N)$ analog is given in Sections 2 and 3.

In a theory with fundamental matter the spectrum also contains baryons. The dual Lagrangian now is modified to accommodate the symmetry of baryon number. This is discussed in Section 4. It turns out that two kinds of solitons are prominent. One type carries one unit of topological charge $Q$ and a baryon number $1 / N$. This is the constituent quark. Another type

\footnotetext{
${ }^{4}$ When fundamental charges are absent, the topological t'Hooft's $Z_{N}$ subgroup of $U_{\Phi}(1)$ remains intact. These models may have another phase in which $Z_{N}$ is unbroken and $\langle V\rangle=0$. This is the Higgs phase in which $Q$ is screened. When fundamental matter fields are present, t'Hooft's $Z_{N}$ symmetry is also broken explicitely. Nevertheless the vortex operator $V$ exists as a local eigenoperator of $\Phi$ and dual description is still useful.
} 
carries $N$ units of $Q$ and no baryon number. It is useful to think about it as a "constituent gluon". A baryon consists of $N$ constituent quarks and one gluon. For $S U(3)$ this gives the $\mathbf{Y}$-shaped string picture of a baryon [7]. The confinement picture of gauge theories with fundamental matter is also very straightforward and simple in the dual description.

In Section 5 we contrast our approach with that of t'Hooft [5] and briefly discuss some implications of these ideas for four dimensional gauge theories.

\section{Topological charge in the Georgi-Glashow model.}

We start with Georgi - Glashow model in $2+1$ dimensions for which the phenomenon of confinement is qualitatively and even quantitatively understood [8]. The Georgi - Glashow model does not contain matter fields in fundamental representation and in this sence is different from QCD. However, as mentioned in the introduction the quark confinement mechanism that we discuss does not differ qualitatively for adjoint and fundamental quarks and therefore it is the simplest model where this mechanism can be seen in action.

The Lagrangian of the model is

$$
\mathcal{L}=-\frac{1}{4} F_{\mu \nu}^{a} F^{a \mu \nu}+\frac{1}{2}\left(\mathcal{D}_{\mu}^{a b} \phi^{b}\right)^{2}+\mu^{2} \phi^{2}-\lambda\left(\phi^{2}\right)^{2}
$$

where

$$
\mathcal{D}_{\mu}^{a b} \phi^{b}=\partial_{\mu} \phi^{a}-i e f^{a b c} A_{\mu}^{b} \phi^{c}
$$


In classical approximation, the model has two phases. For $\mu^{2}>0$ the gauge symmetry is unbroken and the standard confinement phenomenon is therefore expected. For $\mu^{2}<0$ the $S U(2)$ gauge symmetry is broken down to $U(1)$ and the Higgs mechanism takes place. Two gauge bosons, $W^{ \pm}$, acquire a mass, while the third, the "photon", remains massless.

This classical description is only partially correct. The two "phases" are connected analytically [9]. As a result in the continuum limit there is only one phase. This phase is reminiscent of the Higgs phase in that there are electrically charged $W^{ \pm}$constituents and photons. However $W^{ \pm}$are linearly confined and the photon acquires a nonperturbatively small mass. There is no qualitative difference between this "phase" and what one would call the "confinement phase". For example, one can show directly in the continuum limit [8] that the fundamental Wilson loop has the area law behaviour, which is the unambiguous sign of confinement in a theory with only adjoint matter. The confinement of $W^{ \pm}$from the "confinement phase" point of view is just the confinement of the adjoint quarks, since the longitudinal components of the massive vector fields $W^{ \pm}$which appear in perturbative calculations, are precisely the charged degrees of freedom of the quark fields $\phi^{a}$. In the remainder of this section therefore, we do not distinguish between the confinement of $W^{ \pm}$and the confinement of quarks.

The model is useful for our purposes since availability of the dilute monopole gas approximation in the classically "gauge broken" phase makes semiclas- 
sical description of confinement possible [8]. In this section we will develop the picture of confinement described in the introduction using these semiclassical methods in the Higgs - like region of the parameter space of the model. Our hope is, that since there is no phase transition between the Higgs -like and the confinement - like regions of the parameter space, the picture qualitatively remains correct everywhere. If this is the case, our approach, although it does not provide a direct calculational method in the confinement - like region, does provide a clear qualitative understanding of the confinement phenomenon which is the main goal of this paper. We will return to discussion of the confinement region at the end of this section.

The electromagnetic properties of the Georgi - Glashow model are similar to those of Polyakov's compact QED [10]. In compact QED it is clear that the confined charge is just the electric charge. Since in the Georgi - Glashow model the mechanism of confinement is the same, the confined charge should be a $S U(2)$ gauge invariant generalization of the electric charge of compact QED. The generalization which allows the topological interpretation is

$$
\begin{gathered}
J^{\mu}=\frac{1}{e} \epsilon^{\mu \nu \lambda} \partial_{\nu}\left(\tilde{F}_{\lambda}^{a} \hat{\phi}^{a}\right) \\
Q=\int d^{2} x J_{0}(x)
\end{gathered}
$$

where $\hat{\phi}^{a} \equiv \frac{\phi^{a}}{|\phi|}$. It is easy to see, that $W^{ \pm}$indeed carry the topological charge $Q$. In the vacuum $<\hat{\phi}^{1}>=<\hat{\phi}^{2}>=0,<\hat{\phi}^{3}>=1$ (in the unitary gauge). Using the Gauss law eq.(2) one identifies $Q$ as $Q^{3}$ of eq.(3). As in QED the 
current is a curl of local gauge invariant dual field strength $\tilde{f}_{\mu}$.

$$
\tilde{f}_{\mu}=\tilde{F}_{\mu}^{a} \hat{\phi}^{a}
$$

We would like now to express $Q$ manifestly as a topological charge as in eq.(5).

Let us now construct explicitly an operator $V(x)$ in the Hamiltonian formalism. In $\mathrm{QED}_{3}$ the analogous operator has been found in [4].

$$
V(x)=\exp \frac{i}{e} \int d^{2} y\left[\epsilon_{i j} \frac{(x-y)_{j}}{(x-y)^{2}} E_{i}(y)+e \Theta(x-y) J_{0}(y)\right]
$$

where $\Theta(x)=\arctan \frac{x_{2}}{x_{1}}$ is the polar angle. The crucial property of $V$ which ensures that the zeroth component of eq.(可) is satisfied is the term $\int d^{2} y \Theta(x-$ y) $J_{0}(y)$ in the exponent. Clearly the same term must be present in the definition of $V(x)$ in nonabelian case. However this operator by itself is nonlocal. In QED, the second term in eq.(11) completed $V$ to the operator of a singular gauge transformation with gauge parameter

$$
\chi(y)=\frac{1}{e} \Theta(x-y)
$$

This was necessary for $V(x)$ to be a local scalar field. Since $V(x)$ is an operator of a singular gauge transformation, it does not act on any local gauge invariant variable, except possibly at the point $x$ ? . Euclidean Green's functions $<T V\left(x_{1}\right) \ldots V\left(x_{n}\right)>$ in the path integral formalism are given by

\footnotetext{
${ }^{5}$ Note that unlike operators generating regular gauge transformations, in Hamiltonian formalism, the generator of singular gauge transformations does act nontrivially on physical states.
} 
the partition function of the model in the presence of magnetic monopoles

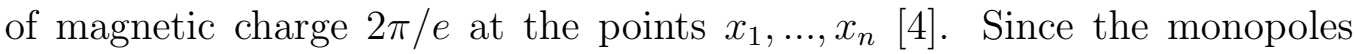
satisfy the Dirac quantization condition, the Dirac strings are invisible and the correlators are Lorentz covariant.

In the nonabelian case the analogous operator of singular gauge transformation is

$$
V(x)=\exp \frac{i}{e} \int d^{2} y\left[\epsilon_{i j} \frac{(x-y)_{j}}{(x-y)^{2}} \hat{\phi}^{a}(y) E_{i}^{a}(y)+e \Theta(x-y) J_{0}(y)\right]
$$

The gauge function is now itself field dependent

$$
\chi^{a}(y)=\frac{1}{e} \Theta(x-y) \hat{\phi}^{a}(\vec{y})
$$

which ensures gauge invariance of $V$. This is the explicit gauge invariant form of t'Hooft's "disorder parameter" [5]. Its locality and Lorentz covariance follow by the same argument as in the abelian case. Since $V(x)$ is a scalar field, not only zeroth but also the spatial components of eq.(付) are satisfied. Therefore $Q$ is the topological charge which counts windings of the phase of $V(x)$.

Our aim now is to write down the low energy effective Lagrangian for the field $V(x)$. We will derive it using dilute monopole gas approximation. The structure of the effective Lagrangian is determined essentially by symmetries of the theory. In QED, apart from obvious Nöther symmetries, a very important symmetry for construction of effective dual Lagrangian has been the magnetic flux symmetry. It is generated by the magnetic flux through the 
plane $\Phi=\int d^{2} x B$. The conservation of the corresponding current $\tilde{F}_{\mu}$ is just the homogeneous Maxwell equation: $\partial^{\mu} \tilde{F}_{\mu}=0$. In the dual Lagrangian this symmetry is represented linearly. The reason is that the operator $V$ is an eigenoperator of the magnetic field:

$$
[V(x), B(y)]=-\frac{2 \pi}{e} V(x) \delta^{2}(x-y)
$$

As in QED, it is easy to check that $V(x)$ eq.(13) is a local eigenoperator of abelian magnetic field $b(x) \equiv \hat{\phi}^{a} \tilde{F}_{0}^{a}$.

$$
[V(x), b(y)]=-\frac{2 \pi}{e} V(x) \delta^{2}(x-y)
$$

In the nonabelian case the flux current $\tilde{f}_{\mu}$ defined in eq.(10) is no longer (classically) conserved [5]. However the following modified current for which $V$ is still an eigenoperator, is classically conserved:

$$
\tilde{F}^{\mu}=\tilde{f}^{\mu}-\frac{1}{e} \epsilon^{\mu \nu \lambda} \epsilon^{a b c} \hat{\phi}_{a}\left(\mathcal{D}_{\nu} \hat{\phi}\right)^{b}\left(\mathcal{D}_{\lambda} \hat{\phi}\right)^{c}
$$

The conservation of the magnetic flux $\Phi \equiv \int d^{2} x \tilde{F}_{0}(x)$ is valid classically but does not survive quantization. The theory contains Euclidean solutions, the t'Hooft - Polyakov monopoles. The elementary monopole is a euclidean configuration with the following asymptotics

$$
\hat{\phi}^{a}(x)=\hat{r}^{a}, \quad \tilde{F}_{\mu}^{a}(x)=\frac{1}{e} \frac{\hat{r}^{a} \hat{r}_{\mu}}{r^{2}}
$$

For this solution

$$
\partial_{\mu} \tilde{F}_{\mu}=\frac{4 \pi}{e} \delta^{3}(x)
$$


and

$$
\Phi(t=\infty)-\Phi(t=-\infty)=\frac{4 \pi}{e}
$$

Quantum mechanically this means that once these configurations are taken into account in the path integral, the magnetic flux is not conserved any more. However, since only configurations with an integer number of monopoles have finite action, the total flux can only change by an integer multiple of $4 \pi / e$. Therefore the discrete flux transformations

$$
U_{k} \equiv e^{\frac{i e}{2} k \Phi}
$$

are still symmetries for integer $k$.

$$
U_{k}(t) U_{k}^{-1}\left(t^{\prime}\right)=e^{2 \pi i k m}=1
$$

As is seen from eqs.(16) and (21), not all of them however act independently on physical Hilbert space. Any one of them is equivalent either to the unit transformation or to $U_{k=1}$ since on the physical states the flux takes only integer values (in units of $2 \pi / e$ ). Therefore the dual Lagrangian should be symmetric under $V \rightarrow-V$ ๆ.

The effective Lagrangian of the Georgi - Glashow model can be obtained directly using dilute monopole gas approximation. Polyakov [8] introduced the dual (electric) potential $\eta$ via

$$
\square \eta=\frac{8 \pi}{e} \rho_{m}
$$

\footnotetext{
${ }^{6} \mathrm{An}$ independent argument for existence of the residual $Z_{2}$ flux symmetry was given by t'Hooft [5].
} 
where $\rho_{m}(x)=\frac{4 \pi}{e} n(x)$ and $n(x)$ is the monopole number density. The effective Lagrangian in terms of $\eta$ is

$$
\mathcal{L}=\frac{e^{2}}{32 \pi^{2}}\left[\left(\partial_{\mu} \eta\right)^{2}+M^{2} \cos \eta\right]
$$

where and $M^{2} \sim \frac{M_{W}^{7 / 2}}{e^{3}} \exp \left(-\frac{4 \pi}{e^{2}} M_{W}\right)$ where $M_{W}$ is the mass of the gauge boson.

The effective Lagrangian eq.(24) can be rewritten in terms of the vortex field $V(x)$ defined in eq.(13). The flux anomaly equation

$$
\partial_{\mu} \tilde{F}^{\mu}=\rho_{m}
$$

and eq.(23) imply that in the approximation in which eq.(24) is valid

$$
\tilde{F}_{\mu}=\frac{e}{8 \pi} \partial_{\mu} \eta
$$

From eq.(24) it then follows

$$
[\eta(x), B(y)]=\frac{4 \pi i}{e} \delta^{2}(x-y)
$$

or

$$
\left[e^{i \eta(x) / 2}, B(y)\right]=-\frac{2 \pi}{e} e^{i \eta(x) / 2} \delta^{2}(x-y)
$$

The operator $e^{i \eta / 2}$ is local, scalar, unitary eigenoperator of the magnetic field $B(x)$ and is therefore identical with $V(x)$ of eq.(13).

We therefore replace $e^{i \eta(x) / 2}$ by $V(x)$ and obtain

$$
\mathcal{L}=\frac{e^{2}}{8 \pi^{2}} \partial_{\mu} V^{*} \partial^{\mu} V+\frac{e^{2} M^{2}}{64 \pi^{2}}\left(V^{2}+V^{* 2}\right)
$$


It is convenient to rescale the field $V$ defining $V^{*} V=\frac{e^{2}}{8 \pi^{2}}$ so that its kinetic term has a standard form

$$
\mathcal{L}=\partial_{\mu} V^{*} \partial^{\mu} V+h\left(V^{2}+V^{* 2}\right)
$$

The residual $Z_{2}$ symmetry is manifest in the lagrangian eq.(30). The appearance of the $U_{\Phi}(1)$ symmetry breaking term leads to important consequences in the spectrum of the theory. As opposed to QED the spectrum is not gapless. Since the coefficient $h$ is small, the lightest excitation of Lagrangian eq. (30) (when $<V>\neq 0$ ) is the phase of $V$ which has a small mass

$$
m=2 \sqrt{h}
$$

The explicit symmetry breaking causes a more dramatic change for topologically charged particles. The theory eq.(30) posesses a topological charge

$$
Q=-\frac{i}{4 \pi} \int \epsilon_{i j} \partial_{i}\left(V^{*} \partial_{j} V-c . c .\right)
$$

Excitations that carry this charge are topological solitons of the field $V(x)$. In as far as eq.(30) describes the low energy physics of the Georgi - Glashow model, these topological solitons must be identified with excitations of the original theory. This identification is quite straightforward. The charge $Q$ of eq. (32) can be rewritten in terms of the original variables using eq.(26) and the connection between $\eta(x)$ and $V(x)$ :

$$
Q=\frac{e}{32 \pi^{2}} \int \epsilon_{i j} \partial_{i} \tilde{F}_{j}
$$


and therefore coincides with the topological charge eq.(99). In the Georgi Glashow model the only light particles that carry $Q$ are $W^{ \pm}$. Therefore the lightest topological defects of the field $V$ in eq.(30) are $W^{ \pm}$. The energy of an isolated defect now is linearly infrared divergent rather than just logarithmically divergent as is the case in $\mathrm{QED}_{3}$. The configuration that forms around the defect is a string rather than a hedgehog (Fig.1). The hedgehog configuration is no longer energetically favored since in such a configuration the phase of the field $V$ is not equal to that of $\langle V\rangle \equiv v$ almost everywhere in space. The energy is therefore quadratically divergent: $E \propto h v^{2} L^{2}$, where $L$ is an infrared cutoff. To minimize the energy for a nonzero winding, the system chooses a stringlike configuration, Fig.1. The phase of $V(x)$ deviates from that of $v$ only within a distance $d$ from the line stretching from the location of the defect to infinity.

It is easy to estimate the energy of such a static configuration. The contribution of the gradient term in Hamiltonian is proportional to $\frac{v^{2}}{d}$ and that of the potential to $h v^{2} d L$. Together

$$
E / L=a \frac{v^{2}}{d}+b h v^{2} d
$$

where $a$ and $b$ are positive numerical factors of order 1 . We now optimize the energy with respect to variation of $d$. Not surprisingly the optimal width comes out as

$$
d \propto \frac{1}{\sqrt{h}} \propto \frac{1}{m}
$$


and the energy per unit length (the sting tension) is therefore

$$
\sigma \equiv E / L \propto v^{2} m
$$

The configuration of a defect and an antidefect has the energy proportional to the distance between them. This is true if the separation between the defect and the antidefect is not too large. When the energy of the string exceeds the energy needed for formation of a soliton - antisoliton pair (the core energy) such a pair will be formed and the minimal energy state will contain two weakly interacting pairs, each held together by a string. This is the same effect which is responsible for perimeter law behaviour of very large adjoint Wilson loops in the standard approach. Nevertheless as long as the distance between a soliton and an antisoliton is not too large, the potential is approximately linear.

The quantity $\sigma$ in eq.(36) pertains to the linear potential between $W^{+}$ and $W^{-}$and is therefore the adjoint string tension. It is interesting to note that the calculation of the fundamental string tension in the framework of the dual Lagrangian eq.(30) gives the value of the same order of magnitude. Let us estimate the vacuum expectation value of the fundamental Wilson loop $W(C)$. The easiest way to do that is by using t'Hooft's dual algebra

$$
\begin{aligned}
W(C) V(x) W^{\dagger}(C) & =-V(x), \quad x \in S ; \\
& =V(x), \quad x \notin S
\end{aligned}
$$

Where $S$ is the area enclosed by the curve $C$. Let us choose the vacuum of the 
dual Lagrangian eq.(30) with $\langle V\rangle=v>0$. Then the state $W(C)|0\rangle$ has the field $V(x)$ negative inside $C$ and positive elsewhere. We now approximate the vacuum state of the phase of the vortex operator by the vacuum of a free massive field of mass $m$.

$$
\mid 0>=\sqrt{N} \exp \left\{-\frac{1}{2} \int \operatorname{dxdy} \eta(x) G^{-1}(x-y) \eta(y)\right\}
$$

with $N$ - the normalisation factor and $G^{-1}=2 v^{2} \sqrt{-\square+m^{2}} \delta^{2}(x-y)$ as follows from eq.(30). Then

$$
W(C) \mid 0>=\sqrt{N} \exp \left\{-\frac{1}{2} \int \operatorname{dxdy}[\eta(x)+\phi(x)] G^{-1}(x-y)[\eta(y)+\phi(y)]\right\}
$$

with

$$
\begin{array}{r}
\phi(x)=\pi, \quad x \in S ; \\
0, \quad x \notin S
\end{array}
$$

Finally we have

$$
\begin{aligned}
<0|W(C)| 0> & =N \int d \eta(x) \exp \left\{-\frac{1}{2} \int \operatorname{dxdy} \eta(x) G^{-1}(x-y) \eta(y)\right. \\
& \left.\left.+[\eta(x)+\phi(x)] G^{-1}(x-y)[\eta(y)+\phi(y)]\right)\right\} \\
& =\exp \left\{-\frac{1}{4} \int \operatorname{dxdy} \phi(x) G^{-1}(x-y) \phi(y)\right\} \\
& =\exp \left\{-\frac{\pi^{2}}{2} v^{2} m S+\text { perimeter contribution }\right\}
\end{aligned}
$$

We find therefore for the fundamental Wilson loop the area law with string tension of the same order of magnitude as the adjoint string tension eq.(36). This result is fully consistent with the lattice calculations [2]. 
The rest of the section is devoted to comments on the dual Lagrangian eq.(30).

i) In this Lagrangian $V(x)$ was assumed to be a " $\sigma$ - model type" field:

$$
V^{*} V=\frac{e^{2}}{8 \pi}
$$

The effective Lagrangian describes however only long distance properties of the model. One expects that when higher derivative terms are taken into account, the "radial" part of $V$ becomes dynamicall. Classically, in the $\sigma$ - model Lagrangian defects are pointlike and their energy is logarithmically divergent in the ultraviolet (the core region).

In the Georgi - Glashow model the self energy of $W^{ \pm}$is finite. In the dual Lagrangian this can be achieved by relaxing the constraint eq.(42). The $\sigma$ model field is replaced by the unconstrained complex field $V$ with large mass of the radial component

$$
\mathcal{L}=\partial_{\mu} V^{*} \partial^{\mu} V+h\left(V^{* 2}+V^{2}\right)+\mu^{2} V^{*} V-\lambda\left(V^{*} V\right)^{2}
$$

The coefficients $\mu$ and $\lambda$ must be such that the expectation value of $V$ is $v^{2}=\frac{e^{2}}{8 \pi^{2}}$. There are now three different scales in the theory: 1) $m$ - the small mass of the phase of $V, 2) v$ - the VEV of $V$ and 3) $M_{\sigma}$ - the large mass of the radial component of $V$. At energies $E \ll M_{\sigma}$ the dynamics of the Lagrangian eq. (43) is the same as that of the $\sigma$ - model. At distances

\footnotetext{
${ }^{7}$ This is reminiscent of low energy chiral Lagrangians [11] in which the field $\sigma$ appears at intermediate energies.
} 
of order $1 / M_{\sigma}$ or less the situation has significantly changed: $M_{\sigma}$ serves as an UV cutoff for low energy physics. The size of the defect is $\sim 1 / M_{\sigma}$ and its self energy $M_{q} \sim v^{2} \ln \frac{M_{\sigma}}{v}$. In fact, even within $\sigma$ - model Lagrangian the $\sigma$ particle appears as a "bound state" due to quantum corrections [12], so it does not have to put in by hand. The only difference would be that $M_{\sigma}$ will depend on the other two parameters. This particular linkage will be anyway spoiled by higher derivative terms and we therefore prefer to use the unconstrained phenomenological Lagrangian eq.(43). In fact the appearance of this scalar particle in the effective dual Lagrangian is a welcome feature. It just represents the scalar Higgs boson which also appears perturbatively in the spectrum of the Georgi - Glashow model and is not confined by nonperturbative effects.

ii) We derived the dual effective Lagrangian in the framework of the dilute monopole gas approximation. Let us now discuss what is the natural extension of the picture presented to the confining region. Since the Higgs and the confining regions are connected analytically the spectrum should be qualitatively the same. Of course there are quantitative differences. The most important one from our point of view is that the lowest energy excitation in the confinement regime is presumably the scalar glueball $/$ rather than a pseudoscalar "photon". However it appears to be still possible to describe the low energy dynamics by the same dual Lagrangian. The reason is that

\footnotetext{
${ }^{8}$ Depending on the mass of the matter field it may be more convenient to think about this state as a scalar meson rather than a scalar glueball.
} 
as it stands the Lagrangian eq.(??dual2) already contains both, the pseudoscalar and the scalar degrees of freedom. The phase of the field $V$ is a pseudoscalar. In the Higgs region it interpolates the massive photon and in the confinement region it interpolates a pseudoscalar glueball. The radial degree of freedom interpolates the scalar Higgs particle and a scalar glueball respectively. In order to describe the confinement regime the coefficients of the Lagrangian must change so that the level crossing between the scalar and the pseudoscalar excitations takes place. This is easily achieved if we take in eq. (43) $2 h+\left|\mu^{2}\right|>0$ and $h>\lambda$. Then the expectation value of the field $V$, the pseudoscalar mass $M_{\eta}$ and the scalar mass $M_{\sigma}$ are given by

$$
v^{2}=\frac{2 h+\mu^{2}}{2 \lambda}, \quad M_{\eta}=8 h v^{2}, \quad M_{\sigma}=8 \lambda v^{2}
$$

The important point is that as in the Higgs phase $v \neq 0$. It is clear on general grounds that this should be the case. If $v=0$ the global $Z_{2}$ symmetry would be broken. In that case the two regions would be necessarily separated by a phase transition. However, as mentioned above the phases are connected analytically and therefore the modes of realization of global symmetries must be the same. The topological current $J_{\mu}$ can still be expressed in terms of $V$, eq.(田). Since $v \neq 0$, the charge $Q$ is never screened (as in the Higgs phase) and is well defined everywhere [13]. Classically then the effective dual Lagrangian admits solitonic configurations. It is very natural to think of these solitons as of constituent quarks in the same way as in the Higgs phase. The energetics of these topological defects will be slightly different. 
To see this first consider the limit $M_{\eta}>>M_{\sigma}$. In this limit it is clearly energetically unfavorable to perform a wind of the angle $\eta$ at those points in space where the radial part of $V$ has large value. What will happen therefore is that at the points where $\eta$ is not constant (inside the flux tube) the radial part of $V$ will be not close to its expectation value but rather close to zero. The contribution of the phase $\eta$ to the string tension will then be negligible. However the radial part will now differ from its VEV along the string inside which $\eta$ winds. The width of the strip inside which the radial part of $V$ varies is given by $1 / M_{\sigma}$. Performing the same rough estimate for the string tension as in the Higgs regime we find

$$
\sigma \propto v^{2} M_{\sigma}
$$

We see therefore that the string tension is dominated by the lightest excitation which in this case is a scalar glueball. In the regime where the scalar and the pseudoscalar masses ar comparable evidently both will contribute to the string tension.

To conclude this section, although we are unable to derive the dual Lagrangian directly in the confining region there is a natural extension of the picture of confinement of constituent quarks. The basic mechanism of confinement is still the same. The constituents are topological solitons. The string tension now is mostly due to the variation of the scalar glueball field but the variation of this field along the flux tube is catalyzed by the necessity to perform a wind of the phase $\eta$. 


\section{Generalization to $\mathrm{SU}(\mathrm{N})$.}

In this section we generalize the analysis to $S U(N)$ gauge theories with adjoint quarks.

In these theories the gauge $S U(N)$ can be classically broken down to variety of subgroups. As in the Georgi - Glashow model, classically there are phase transition lines which are erased by quantum effects. We will have in mind the region of parameters in which the dilute monopole gas approximation is applicable, namely the region in which classically $S U(N) \rightarrow$ $U(1)^{N-1}$ [14], 15]. The Lagrangian is

$$
\mathcal{L}=-\frac{1}{2} \operatorname{Tr} F_{\mu \nu} F^{\mu \nu}+\operatorname{Tr}\left|\mathcal{D}_{\mu} \phi\right|^{2}-U(\phi)
$$

where we use matrix notations $\left(\lambda^{a}\right.$ are traceless hermitian matrices, $\operatorname{Tr} \lambda^{a} \lambda^{b}=$ $\left.\frac{1}{2} \delta^{a b}\right)$

$$
\begin{gathered}
A_{\mu} \equiv A_{\mu}^{a} \lambda^{a}, \quad \phi \equiv \phi^{a} \lambda^{a} \\
F_{\mu \nu}=\partial_{\mu} A_{\nu}-\partial_{\nu} A_{\mu}+i e\left[A_{\mu}, A_{\nu}\right], \quad \mathcal{D}_{\mu} \phi \equiv \partial_{\mu} \phi+i e\left[A_{\mu}, \phi\right]
\end{gathered}
$$

The potential $U$ is chosen in such a way that $\mathrm{VEV}$ of $\phi$ has $N$ nondegenerate

eigenvalues. There are $N-1$ (the rank of $S U(N)$ group) mutually commuting generators which commute with $\phi$. In the basis in which $\phi$ is diagonal these are the diagonal generators

$$
\begin{gathered}
H_{1} \equiv \frac{1}{2} \operatorname{diag}\{1,-1,0 \ldots 0\}, \quad H_{2} \equiv \frac{1}{2 \sqrt{3}} \operatorname{diag}\{1,1,-2,0 \ldots 0\}, \ldots, \\
H_{r} \equiv \frac{1}{\sqrt{2 r(r+1)}} \operatorname{diag}\{1, \ldots, 1,-r, 0, \ldots 0\}, \ldots, H_{N-1} \equiv \frac{1}{\sqrt{2 N(N-1)}} \operatorname{diag}\{1, \ldots, 1,-(N-1)\}
\end{gathered}
$$


These generators define $N-1$ unbroken directions each corresponding to an "electric" charge. Therefore any particle in the theory is characterized by the $N-1$ dimensional vector of eigenvalues: $\vec{q} \equiv\left(q_{1}, \ldots, q_{N-1}\right)$. The charges of gauge bosons are roots of $S U(N)$. The $N-1$ zero roots correspond to (classically) massless "photons", while the charges of massive gauge bosons, $W$, are nonzero roots. Charged $W$ 's are linearly confined, as follows for example from the dilute monopole gas approximation.

It is clear that instead of just one topological charge as in the $S U(2)$ case, here one has to define $N-1$ independent topological charges. The above description is not gauge invariant. We will now construct explicitly the gauge invariant expression for the charges.

Let us start by defining $N-1$ (gauge invariant) analogs of $\tilde{f}_{\mu}$ eq.(10). They will be defined as

$$
\tilde{f}_{r}^{\mu} \equiv 2 \operatorname{Tr} \tilde{F}^{\mu} \hat{\phi}_{r}
$$

where $N-1,(N \times N)$ hermitian traceless matrices $\hat{\phi}_{r}$ satisfy the following conditions:

1) $\hat{\phi}_{r}$ transforms under the gauge group covariantly as an adjoint representation;

2) $\left[\hat{\phi}_{r}, \hat{\phi}_{s}\right]=0$

3) $\operatorname{Tr} \hat{\phi}_{r} \hat{\phi}_{s}=\frac{1}{2} \delta_{r s}$

4) The eigenvalue spectrum of $\hat{\phi}_{r}$ should be identical to that of $H_{r}$ for any $r=1, \ldots, N-1$. 
The matrices $\hat{\phi}_{r}$ can be constructed from a single matter field $\phi$ in the form

$$
\hat{\phi}_{r}=\sum_{n=1}^{N-1} \alpha_{r n} \phi^{n}
$$

where $\alpha_{r n}$ depend only on the invariants $\operatorname{Tr} \phi^{m}$. The first three conditions are satisfied by the set $\hat{\phi}_{r}^{\prime}$ constructed using the elementary Gramm - Schmid algorithm. The only nontrivial condition is 4). To satisfy it we will work in the basis in which $\hat{\phi}_{r}^{\prime}$ are diagonal and the eigenvalues $x_{k}$ of $\hat{\phi}_{1}^{\prime}$ are ordered $x_{1}>x_{2}>\ldots>x_{N}$. Since $\hat{\phi}_{1}^{\prime} \propto \phi$ and the eigenvalues of $\phi$ near the vacuum are all different, this ordering is always possible. In this basis $\hat{\phi}_{r}^{\prime}$ have a form

$$
\hat{\phi}_{r}^{\prime}=O_{r s} H_{s}
$$

where $O_{r s}$ is an orthogonal matrix that depends only on the invariants. The equations determining the matrix elements of $O$ are found taking trace of powers of eq.(51). The solution exists by construction. Having found $O$, we define

$$
\hat{\phi}_{r}=O_{s r} \hat{\phi}_{s}^{\prime}
$$

which satisfies all the requirements.

As an example consider $S U(3)$. In this case

$$
\hat{\phi}_{1}^{\prime}=\frac{\phi}{\sqrt{2 \operatorname{Tr} \phi^{2}}}, \quad \hat{\phi}_{2}^{\prime}=\frac{1}{\sqrt{2\left[-2\left(\operatorname{Tr} \hat{\phi}_{1}^{\prime 3}\right)^{2}+\operatorname{Tr} \hat{\phi}_{1}^{4}\right]}}\left[-2\left(\operatorname{Tr} \hat{\phi}_{1}^{\prime 3}\right) \hat{\phi}_{1}^{\prime}+\hat{\phi}_{1}^{\prime 2}\right]
$$

The orthogonal $2 \times 2$ matrix $O$ is parametrized

$$
O=\left(\begin{array}{ll}
\cos \theta & \sin \theta \\
-\sin \theta & \cos \theta
\end{array}\right)
$$


The resulting equation for $\theta$ is

$$
\sin \theta\left(3-4 \sin ^{2} \theta\right)=4 \sqrt{3} \operatorname{Tr} \hat{\phi}_{1}^{\prime 3}
$$

The system of algebraic equations for $N>3$ is in general complicated. We will not need its explicit solution but only the knowledge that it exists.

The gauge invariant form for the conserved topological currents $J_{\mu}^{r}$ is

$$
J_{\mu}^{r}=\frac{1}{e} \epsilon_{\mu \nu \lambda} \partial^{\nu} \tilde{f}^{r \lambda}
$$

In order to represent $J_{\mu}^{r}$ as manifestly topological we define vortex operators $V_{\vec{g}}$

$$
V_{\vec{g}}(x)=\exp 2 i \sum_{r} g_{r} \int d^{2} y\left[\frac{1}{e} \epsilon_{i j} \frac{(x-y)_{j}}{(x-y)^{2}} \operatorname{Tr} \hat{\phi}_{r}(y) E_{i}(y)+\Theta(x-y) J_{0}^{r}(y)\right]
$$

Those are again singular gauge transformations with gauge functions

$$
\chi_{\vec{g}}(y)=2 \sum_{r} g_{r} \Theta(x-y) \hat{\phi}_{r}(y)
$$

An operator $V_{\vec{g}}$ is local only for values of $g_{r}$ for which a discontinuity of the phase in eq.(57) is an integer multiple of $2 \pi$. This holds if

$$
\sum_{r} g_{r} q_{r}=\frac{k}{2}
$$

with an integer $k$ for any eigenvalue of $Q_{r}$ in the theory. This is the analog of the Dirac quantization condition.

For the theory involving matter in adjoint representation only, the allowed values of $Q_{r}$ lie on the $N-1$ dimensional lattice spanned by the root vectors of 
$S U(N)$. The allowed values of $\vec{g}$ therefore lie on the dual lattice. For $S U(N)$ the dual lattice is spanned by weights of the fundamental representation [14, 15]. Only $N-1$ operators $V_{\vec{g}}$ are independent, all the others being equal to products of integer powers of these $V_{i}$. For convenience we will always choose such a set $V_{i}$ which is symmetric under inversion with respect to a hyperplane perpendicular to the isospin axis (the generator $H_{1}$ ).

For concreteness, in the case of $S U(3)$ the nonvanishing roots are $\vec{r}_{1}=\left(\frac{1}{2}, \frac{\sqrt{3}}{2}\right), \quad \vec{r}_{2}=\left(-\frac{1}{2}, \frac{\sqrt{3}}{2}\right), \quad \vec{r}_{3}=(1,0), \quad \vec{r}_{4}=-\vec{r}_{1}, \quad \vec{r}_{5}=-\vec{r}_{2}, \quad \vec{r}_{6}=-\vec{r}_{3}$

The fundamental weights are

$$
\vec{w}_{1}=\left(\frac{1}{2}, \frac{1}{2 \sqrt{3}}\right), \quad \vec{w}_{2}=\left(-\frac{1}{2}, \frac{1}{2 \sqrt{3}}\right), \quad \vec{w}_{3}=\left(0,-\frac{1}{\sqrt{3}}\right)
$$

The elementary flux operators $V_{1}$ and $V_{2}$ correspond to $\vec{g}_{1}=\vec{w}_{1}$ and $\vec{g}_{2}=\vec{w}_{2}$.

The currents $J_{\mu}^{r}$ are manifestly topological when expressed in terms of $V_{i}$.

$$
\vec{g}_{i} \vec{J}_{\mu}=-\frac{i}{4 \pi} \epsilon_{\mu \nu \lambda} \partial^{\nu}\left(V_{i}^{*} \partial^{\lambda} V_{i}-\text { c.c. }\right)
$$

We now derive the dual effective Lagrangian for $V_{i}$. As in the $S U(2)$ case, the currents $\tilde{f}_{\mu}^{r}$ are not conserved. One can again find a modification which is classically conserved

$$
\tilde{F}_{\mu}^{r}=\tilde{f}_{\mu}^{r}-\frac{4 N(N-1)}{e} \epsilon_{\mu \nu \lambda} \operatorname{Tr} \hat{\phi}_{r} \mathcal{D}^{\nu} \hat{\phi}_{r} \mathcal{D}^{\lambda} \hat{\phi}_{r}
$$

(no summation over $r$ is implied.) The (classical) conservation of $\tilde{F}_{\mu}^{r}$ can be easily checked?. The algebra of the vortex operators $V_{r}$ and the flux

\footnotetext{
9 This is readily seen in the unitary gauge in which $\hat{\phi}_{r}=H_{r}$.
} 
generators $B_{r}$ is

$$
\left[V_{i}(x), \vec{B}(y)\right]=-\frac{4 \pi}{e} \vec{g}_{i} V_{r}(x) \delta^{2}(x-y)
$$

The flux symmetry in our model on the classical level is $U(1)^{N-1}$. It is spontaneously broken with $N-1$ massless photons as Goldstone bosons. Nonperturbative quantum corrections qualitatively change the picture. The symmetry is broken explicitly down to a discrete subgroup and the photons as a consequence become massive. This will be seen directly from the effective Lagrangian derived in the dilute monopole gas approximation.

The monopole solutions of this theory were described in [14, 15. The elementary monopoles have magnetic charge vectors proportional to the roots of $S U(N)$

$$
\vec{m}=\frac{4 \pi}{e} \vec{r}
$$

In the dilute monopole gas approximation the "anomaly equation" for the magnetic flux currents is

$$
\partial^{\mu} \overrightarrow{\tilde{F}}_{\mu}(x)=\sum_{a} \vec{m}_{a} \delta^{3}\left(x-x_{a}\right)
$$

Following the standard procedure we introduce into the path integral fields $\vec{\eta}$ via

$$
\square \vec{\eta}=\frac{8 \pi}{e} \sum_{a} \vec{m}_{a} \delta^{3}\left(x-x_{a}\right)
$$

In terms of the fields $\eta$ the effective Lagrangian is

$$
\mathcal{L}=\frac{e^{2}}{32 \pi^{2}}\left[\left(\partial_{\mu} \vec{\eta}\right)^{2}+\sum_{\alpha} M_{\alpha}^{2} \exp \left(i \vec{r}_{\alpha} \vec{\eta}\right)\right]
$$


where the summation runs over all the $N(N-1)$ nonvanishing roots. The masses $M_{\alpha}$ are $M_{\alpha}^{2} \sim \frac{M_{W}^{7 / 2}}{e^{3}} \exp \left(-\frac{4 \pi}{e^{2}} M_{W}\right)$ where $M_{W}$ is the mass of the gauge boson corresponding to the root $\vec{r}_{\alpha}$. We now reexpress this Lagrangian in terms of the elementary vortex operators $V_{i}$. The simplest way to do this is to relate the fields $\eta_{r}$ to the phases $\chi_{i}$ of the vortex operators. From eq.664) it follows that

$$
\left[\chi_{i}(x), \vec{B}(y)\right]=\frac{4 \pi i}{e} \vec{g}_{i} \delta^{2}(x-y)
$$

The commutation relation of $\eta$ and $B$ follows from eqs.(66,67) and the kinetic term in eq. 68):

$$
\left[\eta_{r}(x), B_{s}(y)\right]=\frac{4 \pi i}{e} \delta_{r s} \delta^{2}(x-y)
$$

Therefore

$$
\chi_{i}(x)=\vec{g}_{i} \vec{\eta}(x)
$$

To invert this relation we use the well known orthogonality relation between the roots and fundamental weights of $S U(N)$. For given independent $N-1$ fundamental weights $\vec{w}_{i}$ there are $N-1$ roots $\vec{r}_{i}$ that satisfy

$$
\vec{w}_{i} \vec{r}_{j}=\frac{1}{2} \delta_{i j}
$$

Hence

$$
\vec{\eta}(x)=2 \vec{r}_{i} \chi_{i}(x)
$$

Substituting this relation into the effective Lagrangian eq.(68) and rescaling the fields $V_{i}$ we obtain

$$
\mathcal{L}=\partial_{\mu} V_{i}^{*} \partial^{\mu} V_{i}+\sum_{\alpha} h_{\alpha} \prod_{i} V_{i}^{2 \vec{r}_{\alpha} \vec{r}_{i}}
$$


In particular for $S U(3)$ and $\vec{g}_{i}$ and $V_{i}$ as chosen in eq.61) we get

$$
\vec{r}_{1}=\left(\frac{1}{2}, \frac{\sqrt{3}}{2}\right), \quad \vec{r}_{2}=\left(-\frac{1}{2}, \frac{\sqrt{3}}{2}\right)
$$

and

$$
\mathcal{L}=\partial_{\mu} V_{i}^{*} \partial^{\mu} V_{i}+h_{1}\left(V_{1} V_{2}^{*}+V_{1}^{*} V_{2}\right)+h_{2}\left(V_{1} V_{2}^{2}+V_{2} V_{1}^{2}+\text { c.c }\right)
$$

The $U(1) \otimes U(1)$ classical flux symmetry is anomalously broken down to its $Z_{3}$ subgroup

$$
V_{1} \rightarrow e^{i \frac{2 \pi}{3} k} V_{1}, \quad V_{2} \rightarrow e^{i \frac{2 \pi}{3} k} V_{2}
$$

This is an example of the $Z_{N}$ "topological" symmetry discussed by t'Hooft [5]. The symmetry $V_{i} \leftrightarrow V_{i}^{*}$ is the standard charge conjugation. In addition to it the Lagrangian possesses the $Z_{2}$ symmetry $V_{1} \rightarrow V_{2}$. This is the charge conjugation symmetry associated with the charge $Q_{1}$. It is present in the dual Lagrangian for any $N$. The reason is that for any root vector $\vec{r}_{\alpha}$ of the form $(a, b, \ldots, c)$ there is a counterpart $(-a, b, \ldots, c)$ and the $\mathrm{W}$ - bosons corresponding to these roots are degenerate.

For $S U(4)$ the corresponding results are the following. The roots and the fundamental weights are

$$
\begin{aligned}
& \vec{r}_{1}=\left(\frac{1}{2}, \frac{\sqrt{3}}{2}, 0\right) \quad \vec{r}_{2}=\left(-\frac{1}{2}, \frac{\sqrt{3}}{2}, 0\right) \quad \vec{r}_{3}=\left(0,-\frac{1}{\sqrt{3}}, \sqrt{\frac{2}{3}}\right) \\
& \vec{r}_{4}=(1,0,0) \quad \vec{r}_{5}=\left(\frac{1}{2}, \frac{1}{2 \sqrt{3}}, \sqrt{\frac{2}{3}}\right) \quad \vec{r}_{6}=\left(-\frac{1}{2}, \frac{1}{2 \sqrt{3}}, \sqrt{\frac{2}{3}}\right) \\
& \vec{r}_{i+6}=-\vec{r}_{i} \\
& \vec{w}_{1}=\left(\frac{1}{2}, \frac{1}{2 \sqrt{3}}, \frac{1}{2 \sqrt{6}}\right), \vec{w}_{2}=\left(-\frac{1}{2}, \frac{1}{2 \sqrt{3}}, \frac{1}{2 \sqrt{6}}\right), \vec{w}_{3}=\left(0,0, \frac{3}{2 \sqrt{6}}\right), \quad \vec{w}_{3}=\left(0,-\frac{1}{\sqrt{3}}, \frac{1}{2 \sqrt{6}}\right),
\end{aligned}
$$


The elementary vortex operators are chosen to correspond to $\vec{w}_{1}, \vec{w}_{2}$ and $\vec{w}_{3}$. The dual Lagrangian is

$$
\begin{gathered}
\mathcal{L}=\partial_{\mu} V_{i}^{*} \partial_{\mu} V_{i}+h_{1}\left(V_{1} V_{2}^{*}+V_{1}^{*} V_{2}\right)+h_{2}\left(V_{1} V_{3}^{*}+V_{2} V_{3}^{*}+\text { c.c. }\right)+ \\
h_{3}\left(V_{1} V_{2}^{2} V_{3}+V_{1}^{2} V_{2} V_{3}+\text { c.c }\right)+h_{4}\left(V_{3}^{2} V_{1} V_{2}+\text { c.c }\right)
\end{gathered}
$$

It again displays t'Hooft's $Z_{4}$ symmetry and both charge conjugations. It is clear that these symmetries remain in the dual Lagrangian for any $N$.

The dual Lagrangian eq.(74) exhibits the same confinement mechanism as the Georgi - Glashow model. When the fields $V_{i}$ acquire nonzero VEV, the solitons are linearly confined. In particular, charged $W$ 's are confined into neutral (with respect to all topological charges) composites. In the $S U(3)$ the gauge bosons corresponding to the roots $\vec{r}_{1}, \vec{r}_{2}$ and $\vec{r}_{3}$ are a defect of $V_{1}$, a defect of $V_{2}$ and a bound pair of $V_{1}$ - defect with a $V_{2}$ - antidefect (as follows from eq.(62)).

\section{Baryons.}

The nonabelian gauge theories with quarks in adjoint representation of the color group are the simplest models exhibiting the topological mechanism of confinement. The most important phenomenon present in QCD but absent in the Georgi - Glashow model is the appearance of baryons. The baryon number is defined only when there are quarks belonging to the fundamental (or other nonzero $N$ - ality) representation of the color group. 
The mesons in the dual representation should be again configurations of defect - antidefect connected by a string. As for a baryon, clearly it should contain $N$ defects each carrying $1 / N$ fraction of baryon number (constituent quark). The topological charge should be neutralized by an (baryon number zero) antidefect with topological charge $-N$. This will require the existence of two types of defects in the theory. We will see that this is indeed the case. The $S U(3)$ baryon in this picture is a $\mathbf{Y}$-shape configuration [7].

We now consider the model which in addition to adjoint fields contains a fundamental multiplet of scalar quarks $\theta^{A}$.

$$
\mathcal{L}=-\frac{1}{2} \operatorname{Tr} F_{\mu \nu} F^{\mu \nu}+\operatorname{Tr}\left|\mathcal{D}_{\mu} \phi\right|^{2}+\left|\mathcal{D}_{\mu}^{A B} \theta^{B}\right|^{2}-U(\phi, \theta)
$$

The dual Lagrangian still contains vortex operators, but the elementary vortex operators now are different. The flux currents are defined by eq.(63). A vortex operator again has a form of eq.(57) but the locality is retained only for a subset of vectors $\vec{g}$. Since the theory now contains fundamental charges the Dirac quantization condition eq.(59) must be satisfied with $\vec{q}$ being weights of the fundamental as well as the adjoint representations. As a result $\vec{g}$ lie on the lattice spanned by roots of $S U(N)$. The $N-1$ elementary local operators $V_{i}$ correspond to $N-1$ independent root vectors. For example in $S U(3)$ we choose $\vec{g}_{1}=\vec{r}_{1}$ and $\vec{g}_{2}=\vec{r}_{2}$ of eq.(60). The dilute monopole gas approximation applied to the present theory gives the same effective Lagrangian eq. (68) in terms of the fields $\vec{\eta}$. The relation between $\vec{\eta}$ and $\chi_{i}$ is modified since $V_{i}$ now carry different values of magnetic fluxes. In terms of 
the new $V_{i}$ we obtain

$$
\mathcal{L}=\partial_{\mu} V_{i}^{*} \partial^{\mu} V_{i}+\sum_{\alpha} h_{\alpha} \prod_{i} V_{i}^{2 \vec{r}_{\alpha} \vec{w}_{i}}
$$

For $S U(3)$

$$
\mathcal{L}=\partial_{\mu} V_{i}^{*} \partial^{\mu} V_{i}+h_{1}\left(V_{1} V_{2}^{*}+V_{1}^{*} V_{2}\right)+h_{2}\left(V_{1}+V_{2}+\text { c.c }\right)
$$

The main difference between eq.(74) and eq. (82) is that in the latter no residual flux symmetry remains. This however, does not change the qualitative picture of confinement.

The derivation is valid in the region of parameter space where the phases of all $N-1$ vortex operators are light variables. The mechanism of confinement remains essentially the same in the case when one of the variables is much lighter than the others. In this case the low energy effective dual Lagrangian should contain only the light vortex field $V$. The topological charges that are carried by the defects of heavy $V_{i}$ are confined at small distances. The only topological charge relevant at low energies is the one that corresponds to the light $V$. This situation is realized in the region of parameters in which classically the $S U(N)$ gauge symmetry is broken down to $U(1)$. From now on we will restrict ourselves to this type of models.

Our goal now is to investigate how to incorporate the baryon number in the dual Lagrangian. Nontopological global symmetries should be represented linearly. This was the case with both charge conjugations in eq.(74) and with flavor symmetries in abelian gauge theories [16]. The vortex field 
$V$ is invariant under baryon number transformations. At the energy scale where baryons appear, one cannot describe physics by effective Lagrangian involving $V$ alone. We introduce therefore a baryon number charged field $W$.

To this end we take a "phenomenological" approach. Interactions of the fields $V$ and $W$ should be such that the elementary defect of the field $V$ carries baryon number $1 / N$. This soliton will represent the constituent (fundamental) quark in our string picture. A necessary condition is that in the core of the defect (where $V$ vanishes) the field $W$ has a nonzero value. Outside the core, $W$ approaches its VEV and thus vanishes (since the baryon number symmetry is not broken spontaneously). The interaction potential should favor the configurations in which for $V$ close to its VEV, $W$ is small whereas for small $V, W$ is nonzero. This is conveniently achieved imposing the constraint四

$$
V^{*} V+W^{*} W=\frac{e^{2}}{8 \pi^{2}}
$$

In order to attach the baryon number to the topological soliton, three derivative terms are necessary. The following Lagrangian satisfies the above requirements

$$
\mathcal{L}=\partial_{\mu} V^{*} \partial^{\mu} V+\partial_{\mu} W^{*} \partial^{\mu} W+h\left(V^{*}+V\right)+\frac{1}{2 \pi N} \epsilon^{\mu \nu \lambda} w^{*} \partial_{\mu} w \partial_{\nu}\left(v^{*} \partial_{\lambda} v\right)
$$

where $w \equiv \frac{W}{\sqrt{W^{*} W}}, v \equiv \frac{V}{\sqrt{V^{*} V}}$. The linear term drives the VEV of $V$ to its maximal value. Consequently due to eq. (84) the VEV of $W$ vanishes.

\footnotetext{
${ }^{10}$ The same effect can be achieved by adding an interaction term $\lambda\left(V^{*} V+W^{*} W-\right.$ $\left.\frac{e^{2}}{8 \pi^{2}}\right)^{2}$. However for long distance properties which we discuss these two alternatives are indistinguishable. We choose the $\sigma$ - model type constraint to simplify the calculations.
} 
We calculate the quantum numbers of the low lying solitons using the zero mode quantization method [17]. Classically there are degenerate static solution of the equations of motion. Let us start with the elementary solitons (a single wind of $V$ ). In fact, there is a one parameter set of degenerate classical solutions. The solutions

$$
V(x)=v_{0}(x), \quad W(x)=w_{0}(x) e^{i \alpha}
$$

have the same energy for any $0<\alpha<2 \pi$ due to the baryon number symmetry. The zero mode $\alpha$ is a slow variable, the only one that has to be treated quantum mechanically[17]. Substituting the solution eq.(86) into the Lagrangian we get

$$
\mathcal{L}=\frac{1}{2} \mu \dot{\alpha}^{2}-\frac{1}{N} \dot{\alpha}+\text { const }
$$

where

$$
\mu \equiv 2 \int d^{2} x w_{0}^{*}(x) w_{0}(x)
$$

The momentum conjugate to $\alpha$ is

$$
p=\mu \dot{\alpha}-\frac{1}{N}
$$

and the corresponding Hamiltonian:

$$
H=\frac{1}{2 \mu}\left(p+\frac{1}{N}\right)^{2}
$$

Since $\alpha$ is an angular variable, $p$ has only integer eigenvalues. 
We first consider the generic case $N>2$. In this case the vacuum of the one soliton sector is nondegenerate: $p=0$. The baryon number current is

$$
J_{\mu}^{B}=i\left(W \partial_{\mu} W^{*}-W^{*} \partial_{\mu} W\right)
$$

In this sector the baryon number is

$$
Q^{B}=\mu \dot{\alpha}=p+\frac{1}{N}
$$

Therefore the elementary soliton has baryon number $1 / N$.

The case $N=2$ is special. The vacuum of the Hamiltonian eq.90) is doubly degenerate: $p=0$ and $p=-1$. The two solitons have baryon numbers $+1 / 2$ and $-1 / 2$ respectively. This degeneracy corresponds to an additional global $S U(2)$ symmetry of the $S U(2)$ gauge model eq. (81) - the "custodial" symmetry:

$$
\theta \rightarrow \theta U
$$

where $\theta$ is a $2 \times 2$ matrix:

$$
\theta \equiv\left(\begin{array}{ll}
\theta_{1} & \theta_{2}^{*} \\
\theta_{2} & -\theta_{1}^{*}
\end{array}\right)
$$

Baryon number is one of the $S U(2)$ generators. Therefore particles always come in pairs with opposite baryon number $\square$.

Now we return to the generic case $N>2$. The elementary soliton with baryon number $1 / N$ evidently represents a constituent quark. However, as

\footnotetext{
${ }^{11}$ To represent faithfully the custodial symmetry in the low energy Lagrangian one should promote the field $W$ to an $S U(2)$ multiplet. This is done in Appendix.
} 
we discussed in the beginning of this section, a consistent picture of baryons require the existence of another elementary soliton with quantum numbers $Q=N, Q^{B}=0$. Let us systematically investigate the quantum numbers in different soliton sectors.

In the sector with $Q=q$, the zero mode Lagrangian is

$$
\mathcal{L}=\frac{1}{2} \mu_{q} \dot{\alpha}^{2}-\frac{q}{N} \dot{\alpha}+\text { const }
$$

The momentum conjugate to $\alpha$ is now

$$
p=\mu_{q} \dot{\alpha}-\frac{q}{N}
$$

The corresponding Hamiltonian is:

$$
H=\frac{1}{2 \mu_{q}}\left(p+\frac{q}{N}\right)^{2}
$$

For $q / N<1 / 2$ the vacuum is $p=0$, while for $1 / 2<q / N<1$ the vacuum is $p=1$. The baryon number is

$$
Q^{B}=\mu_{q} \dot{\alpha}=p+\frac{q}{N}=\left\{\begin{array}{l}
\frac{q}{N} \text { for } q<\frac{N}{2} \\
\pm \frac{1}{2} \text { for } q=\frac{N}{2} \\
-1+\frac{q}{N} \text { for } \frac{N}{2}<q<N
\end{array}\right.
$$

Remarkably, for $q=N$ the soliton has baryon number zero. Moreover this is the only additional independent constituent one needs. We will call it "constituent gluon" since its quantum numbers coincide with those of gauge bosons. All the others can be thought of as composites of the constituent quarks and gluons.

For $S U(3)$ and $S U(4)$ these are given in Tables 1 and $2{ }^{\mathbb{2}}$. 


\begin{tabular}{||c|c|c||}
\hline \hline$q$ & $B$ & composition \\
\hline \hline 1 & $\frac{1}{3}$ & $q$ \\
\hline 2 & $-\frac{1}{3}$ & $\bar{q} g$ \\
\hline 3 & 0 & $g$ \\
\hline
\end{tabular}

Table 1: Quantum numbers of solitons for the $S U(3)$ gauge theory.

\begin{tabular}{||c|c|c||}
\hline \hline$q$ & $B$ & composition \\
\hline \hline 1 & $\frac{1}{4}$ & $q$ \\
\hline 2 & $\pm \frac{1}{2}$ & $q q, \bar{q} \bar{q} g$ \\
\hline 3 & $-\frac{1}{4}$ & $\bar{q} g$ \\
\hline 4 & 0 & $g$ \\
\hline
\end{tabular}

Table 2: Quantum numbers of solitons for the $S U(4)$ gauge theory.

It is interesting to note that the Lagrangian for the theory involving fundamental quarks has the model considered in sections 2 and 3 as its limit. One expects that the mass of the field $W$ becomes large together with the mass of fundamental quarks. Indeed, as the mass of $W$ increases, the region inside the soliton's core in which $W \neq 0$ shrinks. Consequently $\mu$ in eq. (88) decreases as well. From the Hamiltonian eq.(97) it follows that only for $q=N$ (the gluon) the soliton's energy does not diverge in the limit $m_{q} \rightarrow 0$. In this limit $m_{W} \rightarrow \infty$. Therefore the only "survivors" are the solitons with winding $N$ - the constituent gluons. The field $V^{\prime} \equiv V^{1 / N}$ becomes local and in terms of this field we return to the $Z_{N}$ symmetric Lagrangian.

\footnotetext{
${ }^{12}$ Note the degeneracy at $q=N / 2$ for any even $N$. Presumably more accurate treatment lifts this degeneracy since there is no symmetry in eq.(85) accounting for it.
} 
Flavor symmetries can be taken into account in a way similar to that used for baryon number.

\section{Discussion.}

In this paper we proposed a new qualitative picture of confinement in $2+1$ dimensional nonabelian gauge theories. The main idea is that constituent quarks are topological defects of a local field $V$. This field $V$ was explicitly constructed in terms of original Yang - Mills variables. In the nonabelian gauge theories the magnetic flux symmetry $V \rightarrow e^{i \alpha} V$ is explicitly broken and in the confining phase $\langle V\rangle=v \neq 0$. In this situation strings of electric flux are formed between point defects. The topological charge carried by quarks is thereby linearly confined. Baryons in this picture contain $N$ constituent quarks and one "constituent gluon" and for $S U(3)$ have a $\mathbf{Y}$ - shaped form. In the $S U(N)$ gauge theories we identified $N-1$ independent topological charges and $N-1$ corresponding vortex fields $V_{i}$.

The vortex operators $V_{i}$ are closely related to the "disorder parameter" introduced in this context by t'Hooft [5]. It is important to realize, however, that the basic philosophy of t'Hooft's approach is different from ours. t'Hooft proposed to understand the linear confinement as formation of line defects, the Bloch walls, between the different vacua that correspond to different directions of spontaneous breakdown of the residual $Z_{N}$ flux symmetry. He

showed that in terms of the elementary vortex fields defined in a theory 
without fundamentals the wall is attached to any fundamental quark. The linear potential is understood as constant linear energy density stored in this line defect.

There are two major problems with this idea. First, according to t'Hooft, there should be no confinement of adjoint quarks. This contradicts more recent results [2] which show that there is no basic difference between the adjoint and the fundamental string tension. The picture discussed in this paper does not suffer from this problem since it indeed leads to the fundamental and the adjoint string tensions of the same order of magnitude, as shown in Section 2. The second problem with t'Hooft's approach is a certain lack of consistency of the picture itself even for fundamental quarks. It is a mystery where does the Bloch wall end inside a meson. Obviously, the line defect of this kind should either stretch all the way to infinity or form a closed ring. In this picture of a meson, a piece of the line defect is seen clearly, but the rest (including the location of the quarks) is blurred by heavy mist. Indeed, it is impossible to visualize a meson as a well defined configuration of the field $V$, since $V$ defined by t'Hooft, is not a local field in a theory with fundamental quarks.

The difference between t'Hooft's approach and the one advocated in this paper is that t'Hooft emphasizes the importance of line defects (strings) while we concentrate on the picture of quarks (both fundamental and adjoint) as point defects. In a theory with fundamentals the elementary local vortex field 
$V_{\text {fundamental }}$ carries $N$ units of flux compared to $V_{\text {adjoint }}$ in a theory with only adjoint charges. Therefore in our picture the string is not a line defect. It is just a stringlike field configuration which minimizes the energy of a defect antidefect pair and thus necessarily forms when the flux symmetry group is not a continuous one ${ }^{\mathrm{T} 3}$.

It is generally believed that confinement in $2+1$ dimensions is quite similar to that in realistic $3+1$ dimensional nonabelian gauge theories like QCD. Some of the key elements of the above picture can be carried over to $3+1$ dimensions. First, the definition of the topological charge is still given by eq.(9). As in $2+1$, the analogy with the confining phase of $3+1$ dimensional compact QED suggests that this is the correct identification of the confined charge. It would be very interesting to measure the corresponding form factor of hadrons directly by Monte Carlo simulations of the lattice gauge theories. If the identification is correct, the mesons should have large topological dipole moments.

The flux symmetry (which in $3+1$ we prefer to call "magnetic symmetry") has also a straightforward generalization. This was done in [18 for noncompact QED. The conserved current is the dual field strength $\tilde{F}_{\mu \nu}=\epsilon_{\mu \nu \lambda \rho} \partial^{\lambda} A^{\rho}$ [4. The fact that the current is an antisymmetric tensor results in nontrivial

\footnotetext{
${ }^{13}$ The residual flux symmetry could be $Z_{N}$ or could be absent at all, this is not crucial for the phenomenon of linear confinement in this picture.

${ }^{14}$ The magnetic symmetry defined here should not be confused with the symmetry generated by the monopole charge. The latter in QED can be only thought of as a gauge symmetry, since the magnetic monopole charge density must vanish. In contrast, the magnetic symmetry we are talking about is a global symmetry. Its generators are
} 
Lorentz properties of the photon - the Goldstone particle appearing due to its spontaneous breaking.

In the Georgi - Glashow model the analog is

$$
\tilde{F}_{\mu \nu}=\tilde{F}_{\mu \nu}^{a} \hat{\phi}^{a}-\frac{1}{e} \epsilon_{\mu \nu \lambda \rho} \epsilon^{a b c} \hat{\phi}_{a}\left(\mathcal{D}^{\nu} \hat{\phi}\right)^{b}\left(\mathcal{D}^{\lambda} \hat{\phi}\right)^{c}
$$

Classically this current is conserved but quantum mechanically it is broken in the presence of t'Hooft - Polyakov monopoles

$$
\partial^{\nu} \tilde{F}_{\mu \nu}=J_{\mu}^{m}
$$

One can think about this anomaly in the same way we did in $2+1$ dimensions. It is natural to assume that a theory may contain a massless boson only in the presence of a spontaneously broken (global) continuous symmetry. This "reverse Goldstone theorem" has not been proven. Nevertheless in all known cases (in $d>1+1$ ) whenever a massless boson is present, it has been possible to find a spontaneously broken global symmetry. Massless photons in $Q E D_{4}$ also conform to this rule. Assuming for a moment that this folk theorem is true, one is lead to interesting predictions for four dimensional gauge theories. Once the magnetic flux symmetry is explicitly broken, the gauge bosons can not be massless. Furthermore, according to Swieca's theorem [19] if there is no massless boson, the charges must be confined (or screened).

It is well known that this is the case in confining phases of both, compact QED and Georgi - Glashow models. In this phase, according to the dual magnetic fluxes via any infinite two dimensional surface and the charge density is the magnetic field. For discussion see [18]. 
superconductor picture $\llbracket 7,20 \rrbracket$, the monopoles condense in the vacuum. The anomalous right hand side of eq.([100) is therefore large in the vacuum. The effects of the anomaly such as finite mass of gauge bosons and finite string tension can be seen summing over monopole loops in the partition function [21]. The same calculation in the Coulomb phase does not reveal confinement of charges. In this phase the monopoles do not condense. The magnetic symmetry breaking effects are much smaller. However, according to the above naive argument, they should manifest themselves in processes involving creation of virtual monopole - antimonopole pairs. Since in the weak coupling regime the monopoles are very heavy, the probability of these processes is tiny and the resulting string tension is expected to be small.

At this point one should make a delicate distinction between the compact QED (without matter fields) and the Georgi - Glashow model. In QED without matter fields, although $\tilde{F}_{\mu \nu}$ is anomalous, the field strength $F_{\mu \nu}$ is conserved. In fact the theory is dual to the noncompact QED with electric charges [22]. In this case, since there still is a conserved tensor current, $F_{\mu \nu}$, the theory can contain a massless photon. However, since in this theory the broken current is a tensor rather than a pseudotensor, the photon is a pseudovector (unlike in noncompact QED)四.

The Georgi - Glashow model on the other hand contains both, magnetic monopoles and electric charges, so that neither $\tilde{F}_{\mu \nu}$ nor $F_{\mu \nu}$ is conserved.

\footnotetext{
${ }^{15}$ Although parity can be redefined to make the photon a vector, we mean the standard definition of parity in terms of the original fields $A_{\mu}$.
} 
According to this point of view, in the Higgs phase of the Georgi - Glashow model the photon should have a small mass and the electric charges $\left(W^{ \pm}\right)$ should be very weakly confined (or screened) in any nonabelian gauge theory with semisimple gauge group such as GUTs. In fact, Swieca's theorem can be applied to magnetic as well as to electric charges. Therefore in any phase of the theory the possibilities open to electric or magnetic charges are either to be screened or confined.

This contradicts the widely held belief (inspired by perturbation theory) that in the Higgs phase of the Georgi - Glashow model the photon is massless and $W^{ \pm}$are free. However it is not entirely out of the question that the perturbative picture of the Higgs - confinement phase transition is inaccurate. To understand the reason, let us consider QED rather than QCD. There the Higgs - Coulomb phase transition is usually associated with the spontaneous breaking of the electric charge. However the electric charge being topological, does not have a local order parameter and the difference between the two phases is a nonvanishing expectation value of a nonlocal charged operator in the Higgs phase [13]. But in the absence of a local order parameter the Goldstone theorem is not applicable and there is nothing that tells us that the two phases are not analytically connected. In QED this does not happen since

\footnotetext{
${ }^{16}$ There is a remote possibility to define a combination of $\tilde{F}$ and $F$ which is conserved. This is very unlikely since the values of electric and magnetic charges carried by any particle in the theory are in this case not constrained to have a fixed ratio. Even if it is possible to redefine $\tilde{F}_{\mu \nu}$, the photon will not have definite parity and cannot be identified with the massless photon of the perturbation theory.
} 
there is another symmetry (the magnetic symmetry) that is implemented differently in the two phases. However in the $Z_{2}$ gauge theory, for example, which does not have a conserved magnetic flux, the Higgs and the confining phase are indeed connected analytically [13]. The situation in nonabelian gauge theories may be similar in this respect to a $Z_{2}$ gauge theory, in which case one would indeed expect linear confinement of $W^{ \pm}$.

We stress again that our discussion hinges on the validity of the "reverse Goldstone theorem" which has not been rigorously proven. The lattice results on the Georgi - Glashow model at this stage can not rule out this possibility. The photon mass in the Higgs phase was measured in [23 and was found to be consistent with zero. On the other hand the same authors measured the divergence of the magnetic field and found that it also vanishes. This implies that monopoles are unimportant in the region of parameters where the calculation was performed at all scales between the lattice spacing and the size of the lattice. In this case one would indeed expect that both the anomaly of the magnetic symmetry and the photon mass will be undetectable.

We note that these conclusions would not apply to the standard model, which has a gauge group with an invariant abelian subgroup and has therefore a precisely conserved current $\tilde{F}$. Unfortunately there are no adequate analytical tools available to calculate the photon's mass in theories involving both electric and magnetic charges [24]. It would be very interesting to confirm or refute the conclusions based on the present simple minded line 
of reasoning by direct Monte Carlo measurement of the photon's mass and the string tension in the Higgs phase of a $3+1$ dimensional nonabelian gauge theory like the Georgi - Glashow model.

Acknowledgements. We thank G. Gat, E.G. Klepfish and A. Krasnitz for discussions and M. Marcu for correspondence. This work is supported by NSERC of Canada.

\section{Appendix. A Dual Lagrangian with Cus- todial SU(2) Symmetry.}

In this appendix we construct the low energy dual Lagrangian for the $\mathrm{SU}(2)$ gauge theory with fundamental quarks. In addition to the vortex field $V$ the custodial symmetry forces us to introduce the complex doublet $W_{i}, i=1,2$. As in the $\mathrm{SU}(\mathrm{N})$ case we still retain the $\sigma$ - model constraint

$$
V^{*} V+W_{i}^{*} W_{i}=\frac{e^{2}}{8 \pi}
$$

The standard kinetic term for $W_{i}$ has larger symmetry than required: $S O(4)$ rather than $S U(2)$. This symmetry should be reduced by adding another two derivative term of the form

$$
f\left(W^{*} W\right)\left(W^{*} \partial_{\mu} W-W \partial_{\mu} W^{*}\right)^{2}
$$

This term has the symmetry $U(2)$, which is still too large. However if the function $f$ is chosen as

$$
f\left(W^{*} W\right)=\frac{1}{4\left(W^{*} W\right)}
$$


the invariant $U(1)$ subgroup of the $U(2)$, the common phase rotation of $W_{i}$, becomes gauge symmetry. As a result only the $S U(2)$ group is the global symmetry on the physical Hilbert space. To get quarks with correct quantum numbers we also have to add the (U(1) gauge invariant) three derivative term. We thus arrive at the dual Lagrangian

$$
\begin{aligned}
\mathcal{L}= & \partial_{\mu} V^{*} \partial^{\mu} V+\partial_{\mu} W^{*} \partial^{\mu} W+\frac{1}{4\left(W^{*} W\right)}\left(W^{*} \partial_{\mu} W-W \partial_{\mu} W^{*}\right)^{2}+h\left(V+V^{*}\right)- \\
& \frac{1}{8 \pi\left(W^{*} W\right)\left(V^{*} V\right)} \epsilon^{\mu \nu \lambda}\left(W^{*} \partial_{\mu} W-W \partial_{\mu} W^{*}\right) \partial_{\nu}\left(V^{*} \partial_{\lambda} V-V \partial_{\lambda} V^{*}\right)
\end{aligned}
$$

In the sector with topological charge $q$ (the interesting values are $q=1,2$ ) the degenerate static solutions are of the form

$$
V(x)=v^{q}(x), \quad W_{i}(x)=u_{i j} w_{i}^{q}(x)
$$

Here $v^{q}(x), w^{q}(x)=\delta_{i 1} w^{q}(x)$ is a particular solution and $u_{i j}$ is a constant unitary matrix. Substituting eq.(105) into the Lagrangian eq.(104) we get the Lagrangian for zero modes $c_{i}$ (the first column vector of the matrix $u$ )

$$
\mathcal{L}=\mu_{q}\left[\dot{c} \dot{c}^{*}+\frac{1}{4}\left(c^{*} \dot{c}-c \dot{c}^{*}\right)^{2}\right]-\frac{i q}{2}\left(c^{*} \dot{c}-c \dot{c}^{*}\right)
$$

This leads to the following Hamiltonian

$$
H=\frac{1}{\mu_{q}} J^{2}
$$

where $J^{2}$ is the quadratic Casimir of the $S U(2)$ group. The gauge invariance of the lagrangian eq.(106) also results in the constraint

$$
i\left(\pi^{i} c^{i}-\pi^{* i} c^{* i}\right)=q
$$


For $q=1$ the constraint allows only representations with duality 1 . The lightest of those according to the Hamiltonian eq.(107) is the fundamental representation. Thus we get the custodial doublet of quarks as the lowest lying soliton with $q=1$. For $q=2$ the duality must be zero and the lowest excitation is the custodial singlet. This is the gluon (W boson) of the original theory.

\section{References}

[1] F. Close, An Introduction to Quarks and Partons Academic Press, New York, 1979;

[2] J. Greensite and M.B. Halpern, Phys. Rev. D27, 2545 (1983); C. Bernard, Nucl. Phys. B219, 341 (1983); J. Ambjorn, P. Olesen and C. Petersen, Nucl. Phys. B240, 189 (1984); P.H. Damgaard, Phys. Lett. 183B, 81 (1987);

[3] J. Smit and A.J. van der Sijs, Nucl. Phys. B355, 603 (1991);

[4] A. Kovner, B. Rosenstein and D. Eliezer, Mod. Phys. Lett. A5, 2661 (1990); Nucl. Phys. B350, 325 (1991); A. Kovner and B. Rosenstein, Phys. Rev. Lett. 67, 1490 (1991); Phys. Lett. B266, 443 (1991);

[5] G. t'Hooft, Nucl. Phys. B138, 1 (1978);

[6] N.D. Mermin, Rev. Mod. Phys. 51591 (1979), 
[7] G. t'Hooft, Physica Scripta 25, 133 (1982);

[8] A.M. Polyakov, Nucl. Phys. B120, 429 (1977);

[9] E. Fradkin and S.H. Shenker, Phys. Rev. D19, 3682 (1979);

[10] A.M. Polyakov, Phys. Lett. 59B, 82 (1975);

[11] M. Bando, T. Kugo and K. Yamawaki, Phys. Rep. 164, 218 (1988);

[12] I. Ya. Arefyeva, Ann. of Phys. (N.Y.) 117 (1979), 393;

[13] K. Szlachanyi, Comm. Math. Phys. 108, 319 (1987);

[14] N.J. Snyderman, Nucl. Phys. B218, 381 (1983);

[15] S.R. Wadia and S.R. Das, Phys. Lett. 106B, 386 (1981);

[16] A. Kovner and B. Rosenstein, Mod. Phys. Lett. A5, 2733 (1990); A. Kovner and D. Eliezer, Int. J. Mod. Phys. A7, 2775 (1992);

[17] I. Zahed and G. E. Brown, Phys. Rep. 146, 1986, 1;

[18] B. Rosenstein and A. Kovner, Int. J. Mod. Phys. A6, 3559 (1991);

[19] J.A. Swieca, Phys. Rev. D13, 312 (1976); D. Buchholtz and K. Fredenhagen, Nucl. Phys. B154, 226 (1979);

[20] T. Banks, R. Meyerson and J. Kogut, Nucl. Phys. B129, 493 (1977); 
[21] A.M. Polyakov, Gauge Fields and Strings Harwood Academic Publishers, New York 1987;

[22] L. Polley and U.J. Wiese, Nucl. Phys. B356, 629 (1991);

[23] I.H. Lee and J. Shigemitsu, Nucl. Phys. B263, 280 (1986);

[24] M. Blagojevic and P. Senjanovic, Phys. Rep. 157, 233 (1988); 
Figure 1: A stringlike configuration of $V$ that is formed around a pointlike defect. 